\title{
LA PARATRADUCTION DE L'IDENTITÉ DANS LES ESPACES DE TRADUCTION ET D'INTERPRÉTATION EN MILIEU SOCIAL (TIMS)
}

\author{
THE PARATRADUCTION OF THE IDENTITY \\ IN COMMUNITY INTERPRETING \\ AND TRANSLATING
}

\section{Emmanuel Claude Bourgoin Vergondy}

\section{RÉSUMÉ}

Lorsque nous abordons la question de l'identité dans les espaces de la TIMS, nous faisons face à une question essentielle. En effet, la relation avec l'autre est une évidence dans les pratiques de Santé. Qu'en est-il de ces relations, lorsque la diversité culturelle de nos sociétés actuelles redessine les schémas identitaires établis dans les modèles antérieurs ? Dans cet article $^{1}$ nous traiterons la question de l'identité en TIMS grâce à une analyse par niveaux. Ces niveaux sont ceux définis par le groupe de recherche $\mathrm{T} \& \mathrm{P}^{2}$ de l'Université de Vigo. Ainsi, nous commencerons notre analyse par le niveau empirique - ou paratraductif - pour révéler certains des éléments les plus apparents qui accompagnent ou qui entourent la notion d'identité en TIMS. Dans un deuxième temps nous aborderons le niveau sociologique - ou protraductif - dans lequel nous verrons quels sont les relations entre l'espace en générale, les espaces de santé et la notion d'identité. Enfin, nous atteindrons le niveau discursif ou métatraductif - pour comprendre comment les mots d'autres domaines scientifiques prolongent la notion d'identité et la traduisent en d'autres discours qui dépassent le cadre de la TIMS, mais qui servent malgré tout à la pratique professionnelle du traducteur_interprète en TIMS

Mots-clés : paratraduction, identité ; traduction et interprétation en Milieu Social

\section{ABSTRACT}

When we address the issue of identity in TIMS spaces, we face an essential question. Indeed, the relationship with the other is evident in Health practices. What about these relationships, when the cultural diversity of our current societies redraws the patterns of identity established in previous models? In this paper we will deal with the question of identity in TIMS using a level analysis. These levels are those defined by the T\&P research group of the University of Vigo. Thus, we will begin our analysis with the empirical - or paratraductive - level to reveal some of the most apparent elements that goes with or

\footnotetext{
* Universidade de Vigo, Espanhã. ebourgoin@uvigo.es

Orcid: https://orcid.org/0000-0002-4307-8911

1. Pour ne pas alourdir le texte, nous nous conformons à la règle qui permet d'utiliser le masculin avec la valeur de neutre.

2. http://paratraduccion.com/doctorado/
} 
surround the notion of identity in TIMS. In a second step we will approach the sociological - or protraductive - level in which we will see what are the relationships between space in general, health spaces and the notion of identity. Finally, we will reach the discursive - or metatraductive - level to understand how words from other scientific fields extend the notion of identity and translate it into other discourses which go beyond the framework of TIMS, but which nonetheless serve to professional practice of translator-interpreter in TIMS.

Keywords: paratraduction; identity; community interpreting and translating.

\section{INTRODUCTION}

L'identité est, depuis les temps les plus anciens une thématique récurrente de réflexion qui n'est épargnée par presque aucune science. Ainsi les mathématiques y trouvent leur compte : l'identité est une égalité entre deux expressions mathématiques qui est vraie quelles que soit la valeur de leurs variables et s'exprime par exemple par l'équation suivante $(a+b)^{2}=a^{2}+2 a b+b^{2}$. D'un autre côté, pour l'ethnologie, l'anthropologie et la sociologie, c'est aussi un sujet phare. Claude Lévi Strauss disait lors du célèbre séminaire qu'il dirigea sur cette question, que le thème de l'identité intéresse pratiquement toutes les disciplines (LÉVI-STRAUSS, 2010). En outre, dernièrement, la question identitaire se pose aussi en politique et fait, en France notamment, couler beaucoup d'encre et d'argent puisqu'un Ministère de l'identité nationale y a été créé. Masure (2007) y fait référence en ces termes :

La création d'un ministère de l'Identité nationale suffit donc à inquiéter. On peut craindre les pires effets d'une politisation ouverte de cette identité. La promotion, pour la première fois, de ministres « issus de l'immigration » ne change rien à l'affaire. L'orientation donnée par Nicolas Sarkozy à son discours de Dakar ou l'influence assumée de Maurice Barrès par l'un de ses principaux conseillers ont de quoi inquiéter. Elles témoignent d'une vision de la nation et de ce qui existe au-delà de ses frontières digne du XIXe siècle. (MASURE, 2007, p. 50)

Ainsi l'identité intègre les discours politiques, mais on ne fait que lui donner la couleur qu'on lui suppose être l'unique. Alors la discussion se meurt, chacun campe sur ses positions, ne reconnait que ce qu'il veut bien et tout le reste est étranger, autre et non accepté. D’aucun cherche alors, dans ce communautarisme parfois sectaire, un groupe auquel appartenir, des ressemblances, des points communs ou des divergences. On se regroupe sous les couleurs de drapeaux qu' on adopte ou bien auxquels on assigne ou réassigne une idéologie, une politique, un comportement sexuel, etc. On s'identifie aussi en tribus urbaines, avec des marques identitaires qui comme les vêtements, les coiffures, les tatouages. Le constat de l'identité d'aujourd'hui c'est le constat d'une notion ultra démultipliée. La construction 
identitaire de l'homme du XXe et du XXI ${ }^{e}$ siècle se fait dans une jungle d'étiquettes chaque fois plus hétérogène et où il devient difficile de trouver ses repères.

Malgré le constat que nous venons d'établir, sans doute virulent, nous côtoyons dans nos réalités quotidiennes mais aussi et surtout, pour le sujet qui nous intéresse ici, dans nos réalités professionnelles de traducteurs_interprètes ${ }^{3}$ des situations où l'appréhension de l'identité prévaut. Les traducteurs_interprètes en milieu social ${ }^{4}$ nous connaissons l'identité. Nous savons qu'elle est plurielle, changeante et transformante. Il est difficile d'expliquer en pourcentage, courbes et graphiques des expériences uniques donc irrépétibles de l'identité. Le seul espace de la consultation médicale est le témoin de réalités identitaires exprimées de multiples manières. Ces réalités sont une matière essentielle de la pratique professionnelle quotidienne du traducteur interprète en milieu social, parce qu'il les ressent, les traduit et les interprète. Pour le professionnel de la TIMS l'identité est un élément essentiel parfois vital qu'il lui faut comprendre, traduire et interpréter.

Mais est-ce un élément du discours écrit ? Du discours oral ? Est-ce que l'identité ne se trouve pas en marge du texte ? En marge du discours ? La paratraduction est ce qui est marginal pour la traductologie traditionnelle, c'est cette marginalité que nous voulons analyser dans cet article et dans ce recueil pour y trouver la présence de l'identité. La marge est devenue depuis le premier essor de la paratraduction son espace de réflexion. La marge en paratraduction est habitée de tous ces éléments qui font sens à la fois dans le discours (écrit ou oral) de départ et dans le discours (écrit ou oral) d'arrivée, en accord avec les normes, les croyances et les valeurs de deux sociétés données, celle de départ et celle d'arrivée. Il s'établit ainsi, entre ces deux discours, un lien dialectique à différents niveaux : linguistique, culturel, identitaire, sociologique, etc. La paratraduction et son analyse par niveaux offre la possibilité de pouvoir classifier, ordonner et signifier tout ce qui jusqu'alors en traductologie traditionnelle résultait inclassifiable, tout ce qui était hors du discours et qui ne semblait pas y renvoyer. L'analyse paratraductive permet

3. Cette graphie s'inspire de celle utilisée par le Dr. José Yuste Frías concernant le rapport entre texte et image. Cf : YUSTE FRÍAS, J. (2011) «Traduire le couple texte_image dans la littérature pour l'enfance et la jeunesse», Květa Kunešová [ed.] De L'IMAGE à L'IMAGINAIRE. Littérature de jeunesse, Hradec Králové (République Tchèque): Université Hradec Králové, coll. Gaudeamus, ISBN: 978 80-7435-096-2, pp. 36-54, et plus précisément la page 45. Nous avons recours au caractère de soulignement appelé tiret bas « _ » dans notre perspective de placer la traduction et l'interprétation à un même niveau de considération d'exercice professionnel.

4. L'appellation « en milieu social » est propre à la dénomination francophone de cette figure professionnelle de l'interprétation, qui fait écho au community interpreter en anglais et qui regroupe souvent les termes de médiateur, accompagnateur ou interprète interculturel. 
justement de faire dialoguer les éléments de la marge avec le discours et à différents niveaux.

Ce sont les trois niveaux d'analyse de la paratraduction que nous reprenons dans notre article (NOUSS, 2011 apud YUSTE FRÍAS, 2015) développés au cours des sessions de travail qui suivirent le dernier séminaire T\&P donné par Alexis Nuselovici (Nouss) à l'Université de Vigo :

1. Un niveau empirique qui étudie les éléments paratextuels, verbaux et non verbaux (originaires de codes sémiotique comme le visuel et l'auditif), en lien physique ou virtuel avec le texte à traduire ou le discours à interpréter. Ce qui requiert de la part du professionnel l'acquisition de stratégies de traduction différentes, de celles qu'il utilise lorsqu'il travaille exclusivement avec le code linguistique. Ce serait le niveau paratraductif à proprement parler.

2. Un niveau sociologique qui étudie les agents, les normes, les protocoles et les institutions (publiques ou privées) liés au processus traductif et toutes les phases qui y sont mises en ouvre. Nous nous situerions au niveau protraductif.

3. Un niveau discursif qui étudie les discours sur la traduction, ceux qui guident son fonctionnement, la conceptualise et assurent son rôle dans la société. Il s'agit d'un niveau métatraductif.

Par ailleurs, cette analyse par niveau possède en soi un profil très didactique et visuel. En effet, ce nivellement est une ouverture du regard analytique. Il existe entre le niveau empirique et le niveau discursif, une progression vers l'abstraction et à la fois une ouverture vers d'autres champs de connaissance. Ce mouvement expansif nous rappelle souvent d'une part l'ouverture du champ de vision de la caméra photographique ou de cinéma (BOURGOIN VERGONDY, 2019), cette dernière se situant finalement dans une position zénithale mais il renvoie aussi à la progression qui existe dans les méthodes d'apprentissage de langues étrangères. Car dans ces méthodes actuelles, l'apprenant est placé au cœur de son apprentissage. Il agit et interagit dans un objectif de progression et par niveaux, du A1 au B2 et du $\mathrm{C} 1 \mathrm{au} \mathrm{C}_{2}{ }^{5}$. Alors qu'au niveau $\mathrm{A} 1$ il apprend à se présenter et à parler de lui-même et de tout ce qui l'entoure dans son quotidien, au niveau C2 l'apprenant possède des connaissances linguistiques et culturelles qui lui permettent d'écrire et de parler à partir de sujets complexes qui nécessitent parfois un haut degré d'abstraction.

5. Ces niveaux correspondent aux niveaux définis par le Cadre européen commun de référence pour les langues (CECRL) https://www.coe.int/fr/web/common-european-framework-reference-languages 
Nous pensons ici à des textes poétiques, de littérature, à des commentaires sur des créations artistiques en tous genres.

Nous procéderons, dans les prochaines lignes, à la présentation, non exhaustive afin de respecter les exigences éditoriales du présent chapitre, de divers éléments constitutifs de chaque niveau. Nous verrons ainsi que l'identité peut-être un élément visuel, palpable. Elle est aussi interprétable en termes de professions, de nationalités, de législation, d'éthos ${ }^{6}$, de religions, de cultures, de sociologies ou philosophies entre autres. Nous aborderons certains de ces aspects de l'identité afin de pouvoir étayer notre argumentation sur l'importance de la prise en compte de l'identité et de ses différents niveaux de paratraduction dans les espaces de la traduction et de l'interprétation en milieu social (TIMS). La TIMS ne tient ni de la médiation ni de la résolution de conflit (YUSTE FRÍAS, 2013). L'auteur définit ainsi le rôle de la TIMS :

Le recours à la TIMS vient en aide à la médiation lorsque celle-ci se réalise en dehors de tout conflit, ne serait-ce que pour favoriser, renforcer ou simplement établir des liens entre individus. Ainsi, on trouve la TIMS dans la médiation « créatrice » qui vise la création de liens entre individus et dans la médiation « rénovatrice » tendant à améliorer des liens qui sont devenus indifférents à cause de leur relâchement. Sous cet angle exclusif de résolution et de prévention de conflits, que je viens de décrire sommairement, l'interprète ne devrait jamais être totalement confondu avec un médiateur. (YUSTE FRÍAS, 2013, p. 118)

\section{NIVEAU EMPIRIQUE OU PARATRADUCTIF DE L'IDENTITÉ EN TIMS}

Ce premier niveau est sans doute le plus évident à saisir puisqu'il fait très souvent appel aux cinq sens. C'est un niveau où nos différents moyens de perception et notre expérience physique sont à même d'établir une relation directe avec le sujet de cette publication : l'identité.

\subsection{Les éléments paratraductifs du visuel}

La vue est un des premiers sens que nous mettons en œuvre dans l'expérience communicative. Combien d'expressions font référence à la communication silencieuse, lorsque l'on dit qu'un regard parle ou que l'expression d'un visage en dit long... sur ce que l'autre pense mais qu'il ne dit pas. Alors comment l'identité se manifeste-t-elle visuellement?

Nous évoquerons deux types de manifestation visuelle de l'identité. D'une part, les vêtements, car la tenue vestimentaire est généralement un vecteur de

6. Nous employons le mot éthos dans un sens sociologique dans lequel on se réfère aux us et coutumes. 
la manifestation de l'identité de la personne qui les porte. Pour tout patient qui fréquente les espaces sanitaires, il est facile d'identifier le personnel de santé par le port de la blouse. Ainsi quand on parle des hommes et des femmes « en blanc » on sait parfaitement que l'on se réfère au personnel sanitaire : médecins, infirmiers, auxiliaires, administratifs, etc. Si cette manifestation n'est pas révélatrice de données personnelles sur les personnes elle les identifie dans leur activité professionnelle. De leur côté, les soignants, peuvent tout à fait reconnaitre l'identité culturelle d'un patient si celui-ci porte un voile, un sari, une djellabah, un turban qui sont des marques différenciatrices notables lorsqu'elles apparaissent dans une société occidentale comme les nôtres en Europe. C'est un élément qui peut faire supposer au personnel administratif d'un centre de santé primaire que le patient aura peutêtre besoin d'un service de traduction et/ou interprétation.

Toujours dans la perception visuelle, nous trouvons la gestuelle, les mimiques et l'expression corporelle. Ce sont aussi des éléments essentiels dans toute communication voire plus dans une communication à trois où intervient un traducteur interprète. Dans un monde où l'expression corporelle n'est pas universelle (YUSTE FRÍAS, 2011) on se doit de traduire le geste de la culture de départ pour un autre égal ou équivalent dans la culture d’arrivée.

L'acception commune donnée actuellement à la symbolique du pouce, seul doigt levé d'un poing serré, est l'approbation, le succès, la réussite. Or, cela ne vaut pas pour toute l'Europe, car en Grèce (comme d'ailleurs au Moyen-Orient, en Afrique de l'Ouest et en Amérique du Sud), lever le pouce correspond à lever le majeur en France. Par conséquent, ce qui semble être un geste universel positif se trouve être extrêmement offensant dans d'autres cultures. (YUSTE FRÍAS, 2011d, p. 264)

Ainsi le généraliste levant le pouce face à son patient fait peut-être fausse route en pensant que le message de « Tout va bien » sera celui que le patient interprètera. La signification du geste peut être propre à une culture, à un pays voire encore à une région précise, ce qui en fait une question cruciale. Cette signification se présente ainsi comme un élément identitaire d'une culture, d'une nation ou d'un groupe social plus réduit encore. Il faut alors se rendre à l'évidence que les connaissances culturelles en matière d'expression corporelle, de l'interprète en milieu social servent les intérêts d'une communication efficace entre un professionnel de la santé et un patient allophone. En conséquence, ignorer l'expression corporelle en interprétation en milieu social c'est vouer la communication à un échec partiel voire complet. 


\subsection{Les éléments paratraductifs du toucher}

Remise en question de nos jours à cause de la COVID-19, la poignée de main, ou la bise entre personnes intimes sont des gestes habituels dans nos sociétés occidentales. Cependant, ils ne le sont pas forcément dans d'autres sociétés. Voyons l'exemple de la société japonaise où l'on s'incline pour saluer ou bien la culture musulmane où rarement un homme tendra la main à une femme. Une salutation erronée peut porter préjudice à la communication.

Le toucher est un autre élément important en TIMS. En effet, nous devons rappeler ici que le toucher est au centre des gestes de soins. Tous les jours, les médecins, les infirmières, les infirmiers et les aides-soignants (hommes et femmes) pratiquent des gestes qui vont les amener à toucher le patient. Ce sont des rapports physiques qui peuvent engendrer une douleur lorsque le médecin palpe le ventre d'un patient ou quand l'infirmière change un pansement. Ces gestes parfois incompris d'un patient qui ne partage pas la même culture doivent être expliqués et traduits. En outre, le contact physique dans l'espace de santé implique aussi la vulnérabilité du patient. Car dénudé, il expose son intimité à l'autre, à l'étranger. Comment le patient, de quelque culture que ce soit, doit-il accepter et assumer de remettre son intimité, sa nudité à l'aide-soignant ou à l'aide-soignante chargés de sa toilette lorsqu'il est incapable de se mouvoir ? En effet, le conjoint ou un proche ne sont peut-être pas toujours disponibles pour pallier cette difficulté. Mais ce qui est accepté et reconnu au niveau du contact physique dans la médecine occidentale ne l'est pas forcément dans d'autres cultures, dans d'autres conceptions de la médecine.

Voilà pourquoi le traducteur_interprète en milieu social aguerri à l'éthos des différentes cultures et aux langues qu’il maitrise, saura expliquer au professionnel de la santé comment et pourquoi on salue de telle ou telle façon pour pouvoir ainsi conduire la communication dans un échange fluide et cordial.

\section{NIVEAU SOCIOLOGIQUE OU PROTRADUCTIF DE L'IDENTITÉ EN TIMS}

\subsection{Les espaces de l'identité}

\subsubsection{L'espace territorial}

Dans les modèles sociétaux du passé les espaces identitaires étaient relativement bien définis. Car des premiers hommes nomades nous sommes passés à la sédentarisation, à l'occupation durable d'un espace désormais fixé. Le fait 
d'occuper un unique espace durant des générations identifie ceux qui y habitent et les distinguent des autres groupes. Ce sont plus exactement les interrelations que le groupe entretient avec un territoire défini ce qui les identifient par rapport à un autre groupe. Par exemple, le fait d'habiter la campagne et d'y travailler la terre fait d'une personne un paysan. Cette identité s'oppose alors à celle du citadin dont les activités quotidiennes diffèreront complètement. L'espace qui nous entoure au sens large est donc un élément qui participe de notre identité.

À un niveau géographique plus étendu, les définitions frontalières ont transformé l'espace en pays et elles ont permis à ceux qui les habitent de définir leur identité par rapport à ces frontières. On est français, allemand ou italien parce que l'on habite ces espaces nationaux et que l'on en possède une carte d'identité. Voilà un modèle d'appartenance identitaire fondé sur l'espace de résidence et dont le sujet peut donner la preuve grâce à un document officiel. Et par ce fait, le sujet devient alors étranger à tous les autres espaces et aux autres sujets qui les habitent.

Pourtant, de nos jours, la mobilité est à son comble, on ne s'est sans doute jamais autant déplacé que maintenant. Que ce soit pour des raisons professionnelles, pour des raisons de villégiature nous atteignons en un rien de temps l'autre bout du globe. On pourrait croire alors que les restrictions spatiales et les sentiments d'étrangetés ont disparu. En Europe, l'espace Schengen ouvert en 1985 a donné aux européens y résidant la libre circulation. Il serait innocent de croire que l'espace est le même pour tous, même en Europe. La mobilité accrue que nous évoquions n’appartient qu’à une faible partie de l'humanité.

L'espace n'est pas le même pour tous, les droits de mobilité ne sont inscrits sur [une] aucune carte charte égalitaire comme le montre l'admirable film de Nicolas Klotz, La blessure, qui fait impitoyablement défiler une succession d'espaces carcéraux pour les réfugiés africains croyant trouver une liberté : la cellule où sont parqués à Roissy les demandeurs d'asiles, le squat où s'amassent les immigrés, le camion enfermant les travailleurs clandestins. (NOUSS, 2005, p. 52)

De quelle mobilité parle-t-on lorsque l'on traite de la question de la migration ? Si la mobilité interne de l'espace Schengen se définit en termes de libertés, la mobilité entre pays du nord et pays du sud se définit en termes d'obstacles comme ceux cités par Alexis Nouss précédemment. La migration a de nombreuses causes qui ne glorifient en rien l'humanité : guerre, terrorisme, maltraitance, pauvreté, etc. La liste est longue car chaque acte migratoire trouve une raison particulière en chacun des migrants. Auto-condamné à l'exil, le migrant vit des expériences traumatisantes. 
Quelles implications dans la construction identitaire d'un migrant peuvent provoquer ces traumatismes ? C'est ce que cherche à comprendre Mari Rose Moro, professeur de psychiatrie et psychanalyste, et Claire Mestre investies avec l'équipe de l'association MANA à Bordeaux à aider ces patients qui sont confrontés aux implications cliniques de leur propre expérience exilique. Voilà comment Marie Rose Moro parle de leur investissement auprès de ses patients :

Explorer ensuite le voyage dans l'imaginaire des migrants qui le vivent et le transmettent à leurs enfants. Explorer aussi le voyage dans les histoires de vie et les récits cliniques de nos patients migrants et de leurs enfants pour en montrer l'importance, pour comprendre et soigner tous ces voyageurs infatigables et au-delà, pour changer le regard sur leur être et leur parcours ; pour leur faire enfin une place dans la société qui est en train de se construire avec les cul-plombés, c'est ainsi que Victor Hugo nommait les sédentaires. Fous du voyage, ainsi pourrait-on nommer ceux qui bravent les mers et les obstacles pour arriver dans nos pays tempérés trop souvent inhospitaliers et qui tentent même à inscrire l'inhospitalité dans les lois, les règlements et les institutions de notre pays. (MESTRE \& MORO 2005 in MESTRE, 2008, p. 17. C'est nous qui soulignons en caractères gras.)

Privé d'un espace dans lequel s'identifier, le migrant se perd. La perte d'identité, c'est l'octroi à payer dans la mobilité Sud-Nord. Ces espaces dans lesquels le migrant avait placé ses espoirs de bâtir un nouveau futur, de fonder de nouveaux projets, de s'enraciner à nouveau se referment sur eux-mêmes et excluent l’altérité.

\subsubsection{L'espace de santé}

Dans la majorité des pays et des cultures les espaces de santé sont reconnaissables. Ils font partie de l'environnement quotidien. Mais ce n'est pas toujours le cas. Certaines populations vivent éloignées des structures de santé. D'autres encore, ont des croyances ancestrales qui impliquent la pratique de rituels chamaniques et des soins ancrés dans des traditions apparemment immuables.

Exilés volontairement dans nos sociétés occidentales, certains migrants ne reconnaissent pas l'espace de santé comme un référent. En raison de certaines de leurs croyances ils ne peuvent pas s'identifier dans l'acceptation d'une médecine dite moderne. Souvent, certains ne maintiennent un contact avec les centres de santé qu'en cas d'urgence. Les autres maux sont traités avec des onguent et des prières propres à leur culture. En plus du médecin, un sénégalais par exemple, pourra consulter un marabout soit dans le pays d'accueil ou dans le pays d'origine s'il a l'occasion d'y retourner. Il en reviendra avec les remèdes indiqués par le chaman.

Sans traducteur_interprète le migrant aura du mal à comprendre l'espace de santé. Il existe une codification de l'espace de santé qui n'est pas simple à déchiffrer. 
Nous appliquons des codes de couleur à ces espaces qui peuvent ne pas être reconnus comme tels par le migrant. Pour un occidental une croix verte signifie pharmacie et une croix bleue parapharmacie. Peut-on parier que le migrant aura la même conception de cette signalétique ? Sans doute, non. C'est pourquoi l'interprète en TIMS ne se limite pas à tendre des ponts linguistiques. Il est aussi acteur culturel car il connaît et sait transmettre des signifiés culturels aux acteurs du dialogue à trois. Voilà encore une des raisons pour lesquelles, l'exercice professionnel de la TIMS dans les espaces de santé apparaît alors comme essentiel.

\subsubsection{L'espace législatif}

Comme le disait Moro (2008, p. 17), les pays « d'accueil » légifèrent pour « inscrire l'inhospitalité dans les lois, les règlements et les institutions de notre pays. » Parce que l'identité ne se décline pas seulement sous le seul regard d'une appartenance à un espace partagé. Elle se décline aussi en autorisation de séjour, visas et autres papiers jusqu'à l'obtention de la nationalité et de sa matérialisation en carte d'identité. C'est l'administration française par exemple, qui autorise à vivre et travailler sur son sol. Il y a là tout un nombre de démarches lors desquelles le traducteur_interprète en milieu social peut être amené à accompagner les migrants qui entreprennent des demandes de permis de séjour, visas ou de nationalité. Les espaces administratifs sont dès lors souvent fréquentés par des professionnels de la TIMS.

Sur cet autre chemin d'errance administrative dont la durée peut varier énormément selon les cas, le traducteur_interprète transmet à l'écrit et à l'oral différents discours. Il peut être amené à traduire et/ou interpréter à vue tout un ensemble de formulaires et questionnaires. Il traduira et interprétera aussi des récits de vie, peut-être plus concret et douloureux, tout un ensemble de discours qui paratraduisent le vécu du migrant. Un vécu ou des expériences de vie qui ont participé et participent encore parfois dans ce qu'elles ont de plus traumatisant, à sa construction identitaire. C'est parce qu'il traduit et interprète le sujet migrant que le traducteur_interprète en TIMS sait comprendre l'identité aussi en termes d'expérience exilique ou «d'exiliance ». Car à chaque exercice de traduction et d'interprétation en milieu social nous sommes face à une expérience exilique particulière mais « l'exiliance » (NOUSS, 2013) est commune, elle est partagée par tous les autres sujets migrants nous dit Alexis Nouss. Autant que la traduction et l'interprétation, « l'exiliance » est bipolarisée, texte de départ et texte d'arrivée, discours de départ et discours d'arrivée, de ce qui est propre à soi-même au départ face à ce qui est étranger à l’arrivée. 


\section{NIVEAU DISCURSIF OU MÉTATRADUCTIF DE L'IDENTITÉ EN TIMS}

\subsection{L'identité : une approche par le discours métis}

Penser l'identité par le discours métis ou du métissage c'est tout d'abord abandonner toute idée de mélange tel qu'il est habituellement conçu. Il n’y a, dans cette approche de l'identité, aucun renvoi à l'exotisme. Au contraire le métissage comme le conçoivent Laplantine et Nouss, est une façon de comprendre et d'assumer la multiplicité de notre identité. Il nous faut concevoir notre identité comme une composition de différentes facettes dont le processus d'assemblage pourrait s'assimiler au tissage, un phénomène qui « est unique, particulier et trace son propre devenir »(LAPLANTINE et NOUSS, 2011, p. 10). Cet assemblage d'acquis ou de vécus sont autant de représentations de l'individu comme le conçoivent ces mêmes auteurs dans leur exposé sur la notion de métissage.

Le «moi » métis n'étant pas unique ni séparé des autres, n'étant pas un « moi » du tout, n'est personne à proprement parler, mais n'étant personne, il est tous les autres. C'est ce qu'a voulu montrer l'écrivain portugais Fernando Pessoa (1888-1935) -dont le nom signifie précisément «Personne » en portugais- au cours de sa démarche « hétéronymique ». Nous sommes en mesure de comprendre maintenant pourquoi conférer une identité singulière au métissage se révèle absurde. Il n'est plus question de métissage, s'il est trop bien, trop vite identifié. Ma photo d'identité ne saurait être totalement moi; je ne puis accepter cette réduction à une pose, c'est-à-dire à une seule de mes représentations. (LAPLANTINE et NOUSS, 2011, pp. 68-69)

Nous illustrons cette notion de « représentation » grâce au schéma ci-dessous. La spirale qui porte les sphères intérieures représente le devenir de l'individu qui porte et emporte toutes ses autres représentations. Dans notre cas cet individu, dentiste de profession, est aussi à la fois le père de ses enfants, le volontaire d'une $\mathrm{ONG}$, etc. Le devenir que représente la spirale est à la fois cumul et alternance. Car chaque individu passe inexorablement d'une représentation à l'autre dans son quotidien mais il ajoute au cours de sa vie différentes représentations de lui-même. L'individu évolue en allers et retours qui construisent son devenir métis comme « un tissage ou tressage qui ne peut s'arrêter. » (LAPLANTINE et NOUSS, 2011, p. 72). 


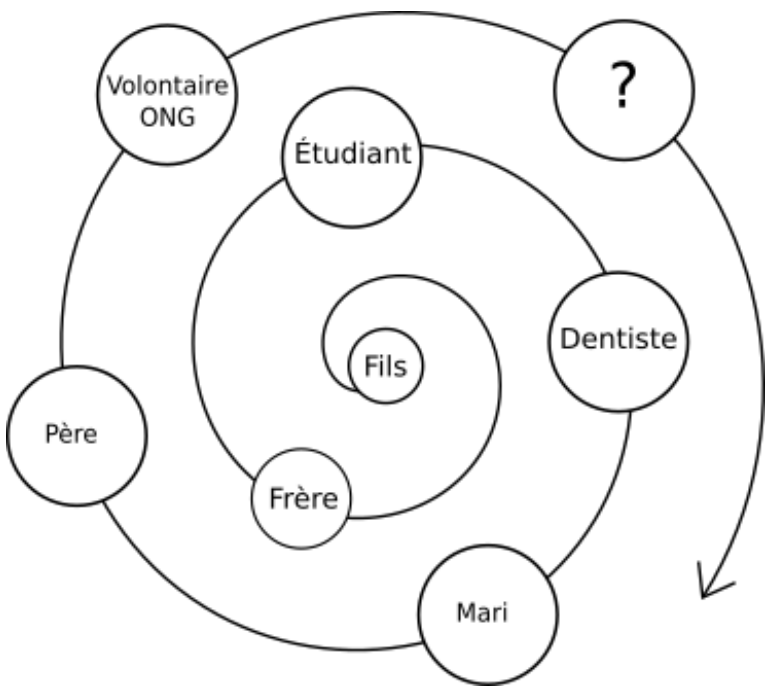

Schéma 1. Illustration des idées développées par Nouss et Laplantine, source : élaboration propre.

Il est somme toute, difficile de figurer un devenir en mouvement par un schéma figé sur une page. Cela semble contradictoire avec la notion même de métissage ou de la pensée métisse :

Si la pensée du métissage est bien une pensée de la médiation qui se joue dans les intermédiaires, les intervalles et les interstices à partir des croisements et des échanges, elle ne saurait se réduire ni au « et », ni à l'entre et à l'entre-deux, qui sont des catégories spatiales. C'est, contrairement au mélange et à la mixité, une pensée de la tension, c’est-à-dire une pensée résolument temporelle, qui évolue à travers les langues, les genres, les cultures, les continents, les époques, les histoires et les histoires de vie. Ce n'est pas une pensée de la source, de la matrice ni de la filiation simple, mais une pensée de la multiplicité née de la rencontre. C'est une pensée dirigée vers un horizon imprévisible qui permet de redonner toute sa dignité au devenir. (LAPLANTINE et NOUSS, 2011, p. 71)

C'est la notion de mobilité, cette tendance au changement, à l'évolution constante de l'individu que nous avons voulu représenter ici par cette spirale qui veut exprimer «la pensée métisse comme pensée de la transformation » (LAPLANTINE et NOUSS, 2011, p. 70), soit le devenir de l'individu. C'est à dire le potentiel de l'individu à intégrer, à échanger avec d'autres individus, avec d'autres sphères, au moyen des expériences vécues dans l'espace, physique et symbolisé en devenir, dans lequel il évolue.

La mobilité se présente dès lors comme un facteur décisif pour le métissage. Il ne s'agit pas seulement d'une mobilité des personnes, qui est certes la plus visible, 
mais aussi une mobilité de la pensée, des idées, de la culture, des langues ou encore du sentiment identitaire. Pour Yuste Frías, la mise en œuvre éthique et politique de la TIMS implique :

une réalisation pratique de la logique du devenir métis. Étant des pensées du lien, de la relation et de la transformation, la traduction et l'interprétation sont surtout des pratiques professionnelles métisses (c'est-à-dire, à la fois métissées et métissantes) et, par conséquent, elles sont bien plus des opérations transculturelles que des procédures interculturelles ou des modalités multiculturelles. (YUSTE FRÍAS, 2014)

La multi-appartenance pour le traducteur_interprète en milieu social doit être un prisme de lecture de l'Autre mais aussi de toutes les individualités qui compose la société. Nous ne pouvons penser l'individu comme unicité car nous sommes le fruit d'un cumul d'identités forgées par « l'exiliance » identitaire.

\subsection{L'identité : une approche par le discours culturel}

L'identité se construit entre autres, dans les rapports qu'entretiennent les sociétés avec la culture. Ces rapports sont uniques et multiples à la fois parce qu'ils sont propres à chacun. C'est-à-dire que notre ressenti culturel, notre relation à la culture s'inscrit dans l'intimité même de notre personne mais en même temps nous pouvons nous identifier à un groupe ou société culturelle dans laquelle nous maintenons le dialogue culturel. Ce qui paraît contradictoire trouve son explication dans ce même dialogue. En effet, lorsque nous partageons nos expériences culturelles, nous les mettons en relation avec d'autres expériences culturelles. Nos sensations peuvent être uniques face à une production artistique mais nous partageons avec d'autres le temps et l'espace culturel. À échanger ainsi nos vécus culturels, nous les mettons en dialogue et nous contribuons par l'échange et le partage culturels à l'assise d'une culture collective, d'une identité culturelle collective. Nous en maitrisons et partageons les codes qu'ils soient linguistiques, sémiotiques, symboliques, etc.

Ainsi les cultures entretiennent un dialogue intrinsèque qui fait à la fois leur singularité et participe de la diversité culturelle mondiale. La relation de la TIMS avec la culture est elle aussi une relation dialoguée, à la différence près qu'il s'agit d'un dialogue entre les cultures. Plus précisément entre deux cultures différentes où le dialogue s'inscrit à la fois dans la différence et dans la reconnaissance qui sont deux positions fondamentalement opposées. La première, lorsqu'on lui autorise un protagonisme exacerbé, finit par détruire le dialogue, tout finit en un monologue du repli sur soi qui s'apparente à un fondamentalisme culturel basé sur le rejet de toute signification de l'étranger. On rencontre à travers l'Histoire nombre d'exemples de 
ces discours des extrêmes tant à gauche qu'à droite - politiquement parlant - qui sont chargés de colère et de haine. Or, « le discours de haine viole l'injonction morale la plus élémentaire d'avoir à respecter la dignité de tout être humain, et ne fait que préjuger de l'infériorité fondamentale des autres. »(TAYLOR, 1994, p. 38). La seconde, celle du dialogue instauré dans la reconnaissance, se distingue en un discours humaniste, parce que comme le dit encore Taylor : « La reconnaissance n'est pas simplement une politesse que l'on fait aux gens : c'est un besoin vital. » (TAYLOR, 1994, p. 42).

Aborder et comprendre le discours de la TIMS et de la Culture est un moyen pour le traducteur_interprète professionnel en milieu social de saisir les nuances des dialogues culturels. Les sociétés culturelles d'antan, qui se suffisaient à ellesmêmes, à la fois étrangères et imperméables ne le sont plus. La mode est désormais à la multiculturalité à l'interculturalité et à la transculturalité : le virus des préfixes atteint de telle façon les discours culturels que l'on finit par ne plus savoir ce qu'est le multi, l'inter ou le trans.

José Yuste Frías (2014) les définit comme des notions « caméléon » trop souvent abordées sans vraiment de questions ni de réflexions sur leur véritable sens. C'est dans son carnet de recherche «Sur les seuils du traduire ${ }^{7}$ » que Yuste Frías propose une réflexion sur chacun de ces trois préfixes à travers la rédaction de trois billets. De façon surprenante mais efficace, il se sert de l'analogie gastronomique pour éclairer le lecteur dans ce foisonnement de préfixations. Il aborde ainsi dans un premier billet sur son blogue de recherche (YUSTE FRÍAS, 2018a), la notion d'interculturalité. Avaler une purée de légumes, c'est selon l'auteur l'image du modèle d'accueil républicain à la française. La France, «terre d'accueil » a ouvert ses portes aux nombreux migrants qui pendant des années ont répondu aux offres de travail du secteur secondaire de l'économie française et se sont installés sur le territoire de la métropole, souvent en périphérie des grandes urbanisations. Mais les exigences de rendement de l'industrie, liées au développement de la robotisation d'une part, ainsi que les différents aléas économiques mondiaux d'autre part, ont freiné l'offre de travail. Peu à peu les politiques d'immigration ont incorporé un contrôle chaque fois plus sévère et des conditions requises d'entrée sur le territoire chaque fois plus strictes. Un exemple cité par José Yuste Frías est celui du CIR (Contrat d'intégration républicaine) « que tout migrant doit signer pour franchir ce que l'Office français de l'immigration et de l'intégration (OFII) désigne, mot pour mot, « la première étape de votre parcours d'intégration en France ». L'intégration à la française suppose l'effacement de toute marque d'identité particulière pour

7. Cf. : https://seuils.hypotheses.org/. 
que le migrant se fonde dans la masse - homogène ? - de la purée de légumes. C'est la citoyenneté républicaine qui doit prévaloir sur toute autre valeur qu'elle soit identitaire ou culturelle. Pour Taylor c'est le reflet d'une culture hégémonique :

Tel qu'on le voit fonctionner, seules les minorités ou les cultures supprimées sont contraintes de prendre une forme étrangère. Par conséquent, la société prétendument généreuse et aveugle aux différences est non seulement inhumaine (parce qu'elle supprime les identités), mais aussi hautement discriminatoire par elle-même, d'une façon subtile et inconsciente. [...] Le libéralisme de la dignité égale semble devoir assumer qu'il existe certains principes universels et aveugles aux différences. (TAYLOR, 1994, p. 63)

Dans son deuxième billet, Yuste Frías (2018b) aborde le concept du multiculturalisme. Les sociétés multiculturelles sont des ensembles composés de sous-ensembles parfaitement identifiables de par leur identité et leur culture. C'est la société américaine faite de «Blacks », «Latinos », etc. Un bol de salade composée comme le dit José Yuste Frías dans lequel, si tout est mélangé, chaque élément est parfaitement identifiable et n'altère en rien, même s'il les jouxte, l'essence même des autres éléments. En TIMS, traduire et interpréter le migrant depuis la perspective multiculturelle, c'est réduire le migrant à une seule appartenance et appliquer sans réflexion profonde, une lecture stéréotypée et donc réduite de l'Autre. Ce qui peut amener le traducteur_interprète en milieu social à une traduction et une interprétation erronée. Enfin, dans le troisième et dernier billet, Yuste Frías (2018c) aborde la transculturalité et s'appuie sur l'analogie gastronomique de la paella pour défendre la diversité culturelle comme une construction identitaire en mouvement « soumise à la multiplicité de ses diverses appartenances ». Traduire et interpréter depuis le prisme de la transculturalité c'est abandonner toute idée de frontière et être disposé à tout moment à se fondre en l'Autre pour conformer « des identités métisses composées de plusieurs appartenance ». C'est l'image de la paella où tous les ingrédients, même s'ils sont reconnaissables, s'imprègnent de la saveur des autres ingrédients et à la fois libèrent la leur.

Dans une mise en œuvre d'une éthique du traduire, le skoppos n'est pas d'intégrer (interculturalité) ni d'assimiler (multiculturalité) le migrant, mais tout simplement, de traduire et d'interpréter le migrant. Traduire et interpréter l'altérité est la meilleure manière d'accueillir le migrant, car c'est en traduisant les différentes appartenances de l'identité de chaque migrant — sans les annuler au presse-purée de l'interculturel ni les survaloriser au buffet à volonté du multiculturalisme que la rencontre entre l'identité de l'un et l'identité de l'autre est possible. (YUSTE FRÍAS, 2018c)

Le traducteur_interprète professionnel en TIMS, conscient de sa propre culture et de celle de celui qu'il traduit et interprète, doit dans sa pratique quotidienne, être toujours disposé à s'investir dans un devenir métis. C'est 
seulement par la compréhension de la multi appartenance identitaire en constante évolution que le professionnel de la TIMS comprendra le potentiel d'une pratique de la traduction et de l'interprétation dans le respect profond de l'Autre, du patient allophone et de son identité multiple.

\section{CONCLUSION}

Nous avons évoqué au début de notre chapitre, l'actualité de la question de l'identité. Nous vivons une époque où l'identité sous différentes acceptions se voit remise en question par une évolution accéléré de notre quotidien. Nos espaces de vie sont changeants, avec la globalisation des échanges et des déplacements, ils deviennent planétaires et par la technologie ils deviennent aussi virtuels. Alors s'il nous est difficile de nous identifier dans ces espaces changeants, nous avons vu que pour un migrant allophone la tâche est sinon plus ardue. Pour le traducteur_interprète qui exerce auprès de ces personnes dans les espaces de santé il est nécessaire de connaître l'autre, autrement dit ce qui fait qu'il est unique et irrépétible (sa culture, son éthos, sa religion). Mais il est tout aussi nécessaire de comprendre comment il est autre. Il faut comprendre les processus de la construction identitaire du migrant, son expérience exilique, l'assimilation de ses traumatismes. Il faut aussi comprendre sa relation aux espaces, le social, la santé, le législatif. Et enfin, il faut aussi qu'il connaisse comment se prolonge la question de l'identité dans d'autres champs de connaissance pour pouvoir s'équiper des outils suffisants pour exercer pleinement sa profession en milieu social. La paratraduction est, nous semble-t-il le moyen d'aborder de façon structurée, grâce à ses trois niveaux d'analyse, une question qui, même situées dans la marge de la traduction et de l'interprétation en milieu social, comporte des implications importantes pour un professionnel de la TIMS. C'est pourquoi la marge et le discours doivent être traités avec la même professionnalité en TIMS.

\section{RÉFÉRENCES}

CASSIN, B. (Dir) (2004). Vocabulaire européen des philosopbies. Dictionnaire des invisibles, Paris: Seuil. LAPLANTINE, F. NOUSS, A. (2011). Le métissage, Paris: Teraèdre.

LÉVI-STRAUSS, C. (2010). L'identité. Séminaire interdisciplinaire dirigé par Claude Lévi-Strauss professeur au Collège de France 1974-1975, Paris: PUF. 
MASURE, F. (2007). État et identité nationale, un rapport ambigu, Journal des anthropologues, Hors-série, pp. 39-50.

MORO, M.-R. (2008). Le voyage est un songe : Détours cliniques, anthropologiques et littéraires, in: Mestre, C. Moro, M-R. [dir.], Partir, migrer: L'éloge du retour, Grenoble: La pensée sauvage, pp.13-24.

NOUSS, A. (2013). Étudier l'exil, halshs-00861243. Accès le 06/04/2020 à https:// halshs.archives-ouvertes.fr/file/index/docid/861243/filename/FMSH-PP-2013-09 Nuselovici.pdf

NOUSS, A. (2005). Plaidoyer pour un monde métis, Paris : Textuel.

TAYLOR, C. (1994). Multiculturalisme, Différence et démocratie, Paris: Aubier.

YUSTE FRÍAS, J. (2018c) Traduire et interpréter le migrant III : partager une paëlla, Sur les seuils du traduire. Un carnet de recherche sur la traduction et la paratraduction, Marseille-Paris-Lisbonne: Hypothèses_OpenEdition_Centre pour l'édition électronique ouverte (Cléo)_CNRS_EHESS_Université d'Aix-Marseille_ Université d'Avignon [en ligne], billet publié le 31/05/2018. Accès le 02/06/2020 à https://seuils.hypotheses.org/1950

YUSTE FRÍAS, J. (2018b). Traduire et interpréter le migrant II : s'attarder au buffet à volonté, Sur les seuils du traduire. Un carnet de recherche sur la traduction et la paratraduction, Marseille-Paris-Lisbonne : Hypothèses_OpenEdition_Centre pour l'édition électronique ouverte (Cléo)_CNRS_EHESS_Université d'Aix-Marseille_ Université d'Avignon [en ligne], billet publié le 07/05/2018. Accès le 11/05/2020 à https://seuils.hypotheses.org/1948

YUSTE FRÍAS, J. (2018a) Traduire et interpréter le migrant I : avaler une purée de légumes, Sur les seuils du traduire. Un carnet de recherche sur la traduction et la paratraduction, Marseille-Paris-Lisbonne : Hypothèses_OpenEdition_Centre pour l'édition électronique ouverte (Cléo)_CNRS_EHESS_Université d'Aix-Marseille Université d'Avignon [en ligne], billet publié le 09/04/2018. Accès le 11/05/2020 à https://seuils.hypotheses.org/1858

YUSTE FRÍAS, J. (2015). Paratraducción: la traducción de los márgenes, al margen de la traducción, Delta, 31, pp. 317-347. Accès le 22/04/2020 http://dx.doi. org/10.1590/0102-445031725373379053 
YUSTE FRÍAS, J. (2014). Interprétation-médiation II : conclusions, Sur les seuils du traduire. Un carnet de recherche sur la traduction et la paratraduction, MarseilleParis-Lisbonne : Hypothèses_OpenEdition_Centre pour l'édition électronique ouverte (Cléo)_CNRS_EHESS_Université d'Aix-Marseille_Université d'Avignon [en ligne], billet publié le 10/06/2014. Accès le 12/04/2020 à http://seuils. hypotheses.org/1223

YUSTE FRÍAS, J. (2013). Aux seuils de la traduction et de l'interprétation en milieu social. In: Benayoun, J.M., Navarro, E. (éds.), Interprétation-médiation : l'an II d'un nouveau métier, Paris: Presses universitaires de Sainte Gemme, pp. 115-145.

Recebido: 17/6/2020

Aceito: 21/7/2020

Publicado: 29/7/2020 